\title{
MEMS based Hand Gesture Wheel Chair Movement Control for Disable Persons
}

\author{
V Sundara Siva Kumar ${ }^{*}$, G.Ramesh ${ }^{\dagger}$ and P Nagesh ${ }^{\ddagger}$ \\ †Department of Electronics \& Instrumentation, Rajeev Gandhi Memorial Engineering College, Nandyal, India \\ ‡Sree Kavitha Engineering College Khammam, India \\ Accepted 22 May 2015, Available online 25 May 2015, Vol.5, No.3 (June 2015)
}

\begin{abstract}
This project is to develop a wheel chair control which is useful to the physically disabled person with his hand movement or his hand gesture recognition using MEMS technology. The use of powered wheel chair with high navigational intelligence is one of the great steps towards the integration of severely physically disabled people. Driving wheel chair in domestic environments is a difficult task for people with arm or hands impairments.The wheel chair is developed to overcome the above problem described above allowing the end users to just perform safe movements and accomplish some daily life important tasks.
\end{abstract}

Keywords: MEMS, Powered wheel chair

\section{Introduction}

The main aim of this paper is to controlling a wheel chair and electrical devices by using MEMS. MEMS accelerometer sensor is micro electro-mechanical sensor which is a highly sensitive sensor and capable of detecting the tilt. This sensor finds the tilt and makes use of the accelerometer to change the direction of the wheel chair depending on tilt. For example, if the tilt is to the right side then the wheel chair moves in right direction or if the left side then wheel chair left direction. Wheel chair movement can be controlled in forward. This project makes use of microcontroller, which is programmed, with the help of Embedded C instructions. This microcontroller communicates with the ADC and motor driver. The MEMS Accelerometer Sensor based sensor detects tilt and provides the information to the microcontroller and the controller judges whether the instruction is right movement or left movement instruction and controls the direction respectively. The controller is interfaced with two dc motors to control the direction of the wheel chair. To perform the task the controller is loaded with intelligent program written using Embedded $\mathrm{C}$ language.

\section{Our proposed work}

\section{3-axis Accelerometer sensor}

This small and highly sensitive accelerometer can detect acceleration, inclination and vibration by

*Corresponding author: V Sundara Siva Kumar

DOI: http://Dx.Doi.Org/10.14741/Ijcet/22774106/5.3.2015.48 measuring the motion in the $x^{-}, y^{-}$, and $\mathrm{z}$-axis simultaneously. By sensing the mounting angle, the sensor can assist in compensating for the devices mounting angle, and here for ekes it possible to use normal SMD technology in high density boards, and also to realize the precise detection of the inclination angle. An interface IC within the sensor package also has temperature sensing and self-diagnosis functions.

\section{Principle of detection}

Piezo Resistor

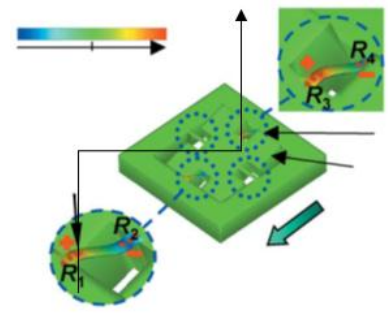

Acceleration along $\mathrm{x}-(\mathrm{y}-)$ axis

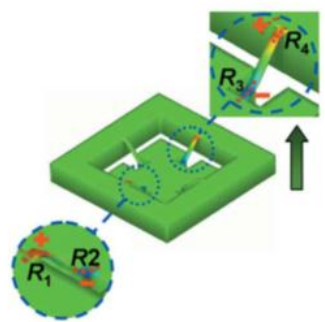

Acceleration along z-axis
Figure1 Principle of Detection

The MEMS 3-axis accelerometer consists of a Mass at the centre of the sensor's chip, which is suspended by 4 Beams doped with Piezo resistive material. When the sensor is subjected to acceleration in any direction, the movement of the Mass causes the 4 Beams to deform and so change the resistance in the piezo material. This enables the sensor to detect the acceleration motion 


\section{Related Work and Motivation}

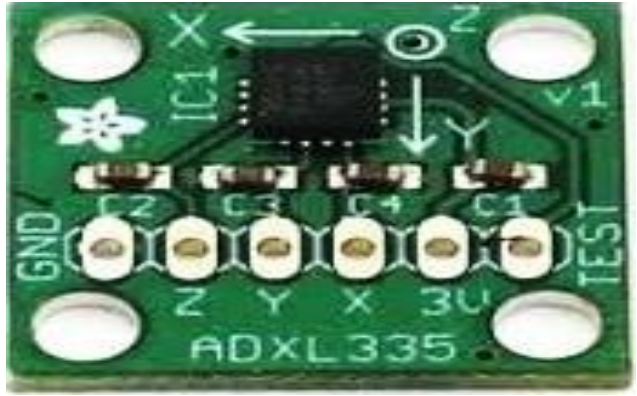

Figure2 MEMS Sensor

The ADXL335 is a small, thin, low power, complete 3axis accelerometer with signal conditioned voltage outputs. The product measures acceleration with a minimum full-scale range of $\pm 3 \mathrm{~g}$. It can measure the static acceleration of gravity in tilt-sensing applications, as well as dynamic acceleration resulting from motion, shock, or vibration. The user selects the bandwidth of the accelerometer using the $\mathrm{CX}, \mathrm{CY}$, and CZ capacitors at the Xout, Yout, and Zout pins. Bandwidths can be selected to suit the application, with a range of $0.5 \mathrm{~Hz}$ to $1600 \mathrm{~Hz}$ for the $\mathrm{X}$ and $\mathrm{Y}$ axes, and a range of $0.5 \mathrm{~Hz}$ to $550 \mathrm{~Hz}$ for the $\mathrm{Z}$ axis.

The ADXL335 is available in a small, low profile, 4 $\mathrm{mm} \times 4 \mathrm{~mm} \times 1.45 \mathrm{~mm}, 16$-lead, plastic lead frame chip scale package.
Micro Electro Mechanical Systems (MEMS) is the integration of mechanical elements, sensors, actuators, and electronics on a common silicon substrate through micro fabrication technology. An accelerometer is an electromechanical device that measures acceleration forces. MEMS accelerometer is a single chip with small size and low cost. Because of their small size and weight, accelerometers are attached to the finger tips and back of the hand. In this model we are usingMMA760FC accelerometer, which is 3axis accelerometer and gives digital output (I2C).

\section{Simulation implementation}

This project is implemented using following software's:

- Keil software - for compilation part

- Proteus 7 (Embedded C) - for simulation part

\section{Keil software}

Keil Micro Vision: is free software which solves many of the pain points for an embedded program developer. This software is an integrated development environment (IDE), which integrated a text editor to write programs, a compiler and it will convert your source code to hex files too.

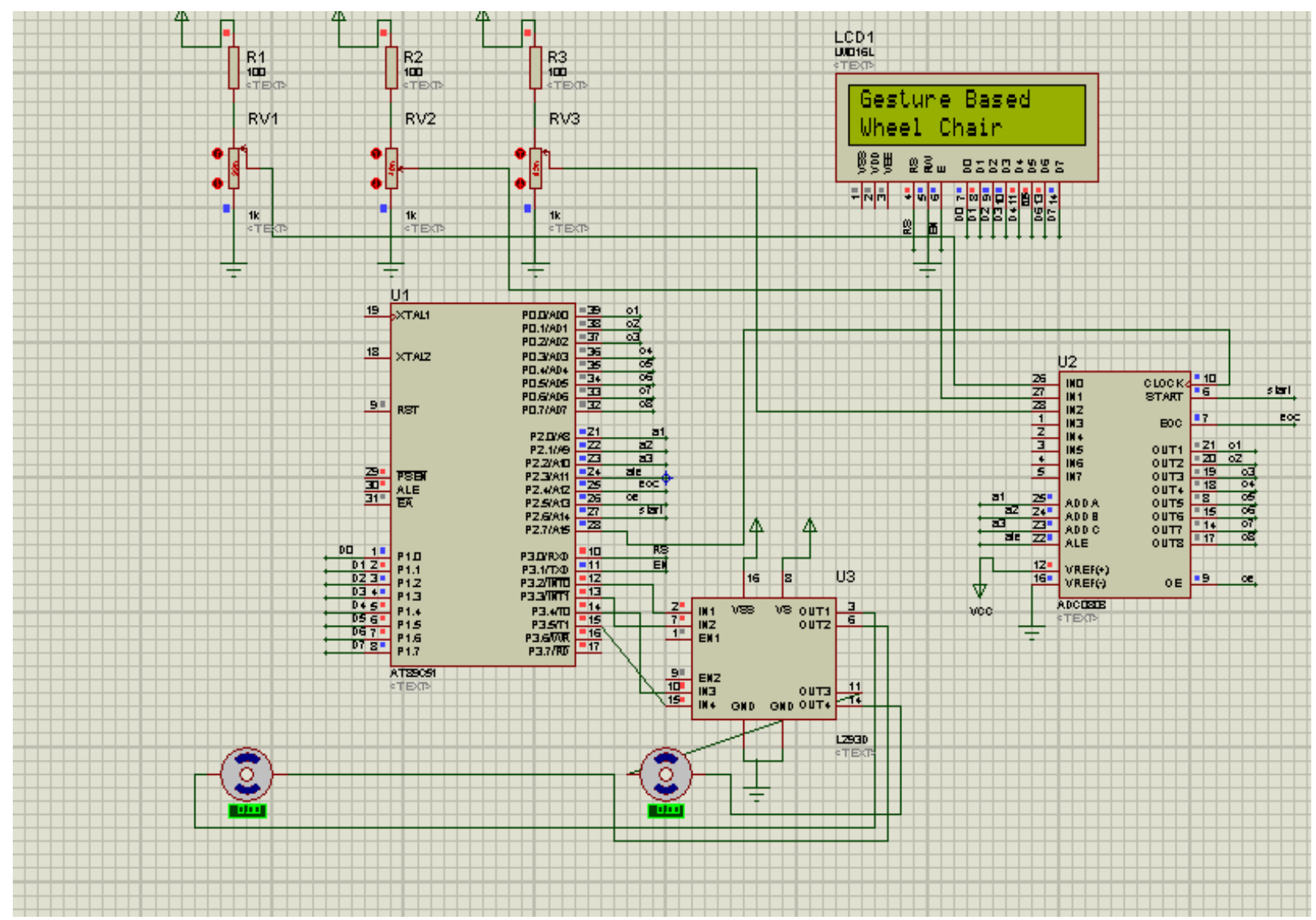

Figure3. Simulation Implementation 


\section{Proteus}

Proteus is software which accepts only hex files. Once the machine code is converted into hex code, that hex code has to be dumped into the microcontroller and this is done by the Proteus. Proteus is a programmer which itself contains a microcontroller in it other than the one which is to be programmed. This microcontroller has a program in it written in such a way that it accepts the hex file from the keil compiler and dumps this hex file into the microcontroller which is to be programmed. As the Proteus programmer requires power supply to be operated, this power supply is given from the power supply circuit designed and connected to the microcontroller in proteus. The program which is to be dumped in to the microcontroller is edited in proteus and is compiled and executed to check any errors and hence after the successful compilation of the program the program is dumped in to the microcontroller using a dumper.

The working procedure of the project is when, the change in the hand gesture then the MEMS generates analog signal from mechanical signal and this is given to ADC which, converts analog to digital signal. Microcontroller doesn't understand analog signal so the digital signals are given to it. According to the change in direction in the MEMS sensor the micro controller controls the motor direction by motor driver.

6. Hard ware Implementation: The components involved in the paper are
1) MEMS sensor
2) $\mathrm{ADC}(0808)$
3) Micro controller(AT89S52)
4) H-bridge(L293D)
5) DC motors

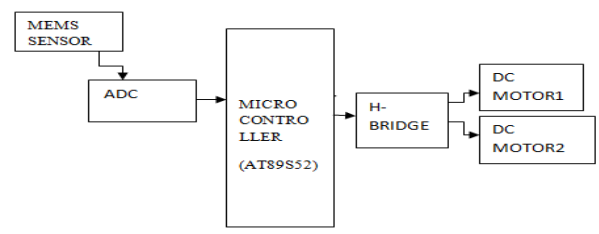

Figure 4 Block Diagram of MEMS Sensor

The above system represents, any change in the direction of MEMS sensor in X-and Y-axis directions then this MEMS sensor has piezo resistive material at the center of the chip, which is suspended by 4 beams doped with piezo resistive material. When the sensor is subjected to acceleration in any direction, the movement of the mass causes the 4 beams to deform and so changes the resistance of the piezo material.
This enables the sensor to detect the acceleration motion. MEMS sensor contain Tilt register. When the changes in the direction, the tilt register values are changed and that values are given to $A D C$, which converts analog to digital values this values are given to microcontroller. Depending on the direction of the MEMS, microcontroller controls the wheel chair directions like LEFT, RIGHT, FRONT, and BACK By implementing the above circuit, the obtained values are:

Table 1 Experimental Values

\begin{tabular}{|c|c|c|}
\hline position & X-axis & Y-axis \\
\hline Left & $>150 \& \&>200$ & $<200 \& \&<250$ \\
\hline Right & $>100 \& \&>200$ & $>150 \& \& 200$ \\
\hline Forward & $>150 \& \&>200$ & $<200 \& \&<250$ \\
\hline Backward & $>100 \& \&>200$ & $<150 \& \&<250$ \\
\hline
\end{tabular}

\section{Conclusion}

This paper is implemented using various components, the project is just a proto type if we make this project as commercial project, then definitely useful to all the disabled people, who are unable to move and unable to drive normal wheel chair their own. With their hand movements they can move wheel chair right, left, front, and back directions with 3-axis accelerometer(MEMS SENSOR) which is a highly sensitive sensor and capable of detecting the tilt. The future scope of the project can be extended using wireless technology, and intelligent hand gesture wheel chair.

\section{References}

(14-16 July 2008), A Wearable Head- Mounted Sensor Based Apparatus for Eye Tracking Applications IEEE International Conference on Virtual Environments, Human-Computer Interfaces, and Measurement Systems Istanbul, Turkey.

(2007) 4 International conference on Electrical and Electronics Engineering (ICEEE 2007) Mexico City, Mexico. September 5-7,

Marhic, B. (2005) Robotic assistance: an automatic wheelchair tracking Intelligent Robots and Systems, (IROS2005). 2005 IEEE/RSJ.

Yi Zhang, Jiao Zhang, Yuan Luo (2011), A Novel Intelligent Wheelchair Control System Based On Hand Gesture Recognition, Proceedings of the 2011 IEEE/ICME International Conference on Complex Medical Engineering May 22-25,Harbin,China,pp:334- 339,201.

Wen J .Li (May, 2012), MEMS Accelerometer Based Nonspecific-User Hand Gesture Recognition. IEEE SensorsJournalVol12, No.5.pp:1166-1173.

Wheel Chair Motion Control Based On Hand Gesture Recognition IJIRTS volume2 No2 